\title{
Neue Mobilitätskonzepte und autonomes Fahren: Potenziale der Veränderung
}

\author{
Barbara Lenz, Eva Fraedrich
}

\section{Inhaltsverzeichnis}

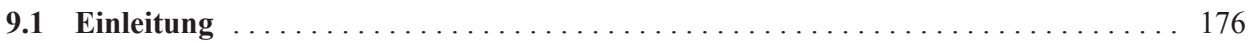

9.2 Carsharing: „Kernapplikation“ neuer Mobilitätskonzepte . . . . . . . . . . . 177

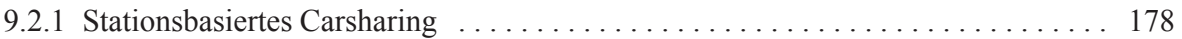

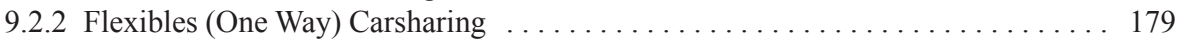

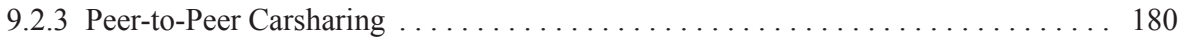

9.3 Nutzer und Nutzung der neuen Mobilitätskonzepte $\ldots \ldots \ldots \ldots \ldots \ldots \ldots \ldots \ldots$

9.3.1 Nutzer und Nutzungsvoraussetzungen . . . . . . . . . . . . . . . . . . . 181

9.3.2 Der Carsharer - der „,neue Mensch“ in einer Sharing Economy? . . . . . . . . . 181

\subsection{Digitalisierung der Alltagswelt als Grundvoraussetzung}

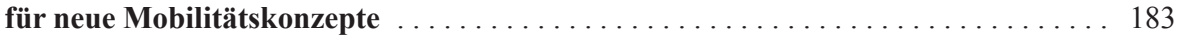

9.5 Weiterentwicklung der neuen Mobilitätskonzepte

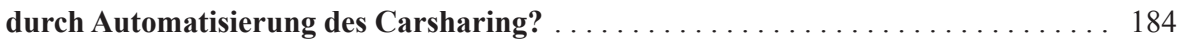

9.5.1 Autonomes Valet-Parken im Carsharing . . . . . . . . . . . . . . . 185

9.5.2 Carsharing unter Einsatz von „Vollautomaten mit Verfügbarkeitsfahrer“ . . . . . 186

9.5.3 Das Carsharing-Fahrzeug als Vehicle-on-Demand .................. 187

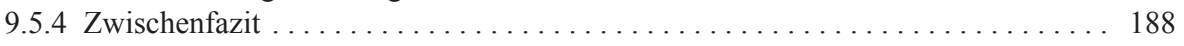

B. Lenz $(\triangle)$

Deutsches Zentrum für Luft- und Raumfahrt e.V., Institut für Verkehrsforschung, Deutschland Barbara.Lenz@dlr.de

E. Fraedrich

Humboldt-Universität zu Berlin, Geographisches Institut, Deutschland eva.fraedrich@geo.hu-berlin.de 


\subsection{Neue Mobilitätskonzepte jenseits von Carsharing:}

Hybridisierung des öffentlichen Verkehrs? . . . . . . . . . . . . . . . . . . . 189

9.6.1 Neugestaltung von Intermodalität und Flexibilisierung des öffentlichen Verkehrs . 190

9.6.2 Individualisierung des öffentlichen Verkehrs . . . . . . . . . . . . . . . . . 191

9.6.3 Verdichtung der Bedienungsmöglichkeiten mit öffentlichem Verkehr . . . . . . . 192

9.7 Implementierung von neuen Mobilitätskonzepten mit autonomen Fahrzeugen … 192

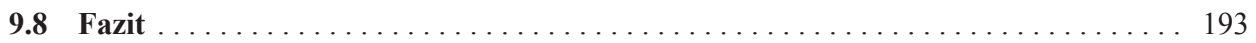

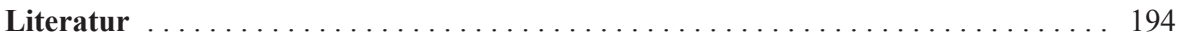

\subsection{Einleitung}

Verkehr ist Ausdruck der Befriedigung von Mobilitätsbedürfnissen mit unterschiedlichen Verkehrsmitteln - im Alltagsverkehr sind Menschen zu Fuß, mit dem Fahrrad, den Verkehrsmitteln des öffentlichen Verkehrs oder dem Pkw unterwegs. Dabei werden zwei große Gruppen unterschieden: Personen mit einer ausgeprägten Präferenz für die Nutzung des privaten Pkw einerseits und Personen mit einer Vorliebe für den sogenannten „Umweltverbund“, d.h. für die Kombination aus öffentlichem Personenverkehr (ÖPV), Fahrradund Fußwegen, andererseits [1]. Zusätzlich bildet sich seit einigen Jahren die Gruppe der „Multimodalen“ heraus, die nicht mehr auf ein spezifisches Verkehrsmittel oder einen bestimmten Verkehrsmittelmix ausgerichtet sind, sondern in ihrem persönlichen Repertoire der Verkehrsmittelnutzung eine große Bandbreite aufweisen [1, 2]. Dieser allmähliche Verhaltenswandel fällt mit der Entwicklung neuer Mobilitätskonzepte zusammen, die zum einen Weiterentwicklungen des klassischen Carsharing darstellen [3], zum anderen etablierte Mitfahrgelegenheiten um neue Formen ergänzen. $\mathrm{Zu}$ den bereits realisierten neuartigen Konzepten gehören flexible Carsharing-Flotten wie die von Car2Go, DriveNow oder Multicity, die als Mobilitätsdienstleistungen in Großstädten in Deutschland, aber auch in zahlreichen Städten Europas und der USA zur Verfügung stehen. Parallel dazu entwickeln sich sogenannte Peer-to-Peer-Angebote, in denen private Fahrzeugbesitzer über eine Internet-Plattform ihr Fahrzeug für eine Mitglieder-Community bereitstellen. Auf Internetplattformen wie Mitfahrzentrale oder Zimride bieten Privatpersonen Mitfahrgelegenheiten auf Strecken und zu Zeiten an, auf bzw. zu denen sie selbst ohnehin unterwegs sind. Zusätzlich etablieren sich derzeit auch mehr und mehr Angebote, wie z. B. Uber oder Lyft, bei denen die Unterscheidung zwischen (semi-)professioneller Personenbeförderung, vergleichbar mit einer Taxidienstleistung, und „klassischer“ Mitfahrgelegenheit nicht immer einfach ist. Die neuen Formen von Carsharing und Mitfahrdiensten entstehen vor allem in den großen Städten und Metropolregionen der Industrieländer.

Das Neue und gleichzeitig auch das Besondere an solchen neuen Mobilitätskonzepten besteht in dem hohen Maß an Flexibilität, das diese Konzepte den Nutzern bieten. Fahrzeuge des flexiblen Carsharing sind ohne Vorplanung zu einem beliebigen Zeitpunkt und 
für eine beliebige Dauer verfügbar. Ähnlich flexibel sind die neuen Mitfahrdienste, sie gleichen dabei allerdings dem konventionellen Taxi. Eine wesentliche Voraussetzung für die Entstehung aller neuen Mobilitätskonzepte sind die Möglichkeiten, die heute hinsichtlich der Vernetzung von Fahrzeugen, Nutzern und Betreibern mithilfe von Informationsund Kommunikationstechnologien vorhanden sind. Dadurch wird ein grundsätzlich einfacher und schneller Zugriff auf Fahrzeuge oder Dienste mittels Internet oder SmartphoneApp überhaupt erst möglich. Dennoch bleibt grundsätzlich der Zugang im Sinne der physischen Distanz zwischen dem Standort des Nutzers und dem des Fahrzeugs als Nutzungshürde bestehen, insbesondere in Gebieten, in denen die Fahrzeugdichte nicht sehr hoch ist.

Mit der Einführung von autonomen Fahrzeugen erscheint es möglich, die bestehenden Konzepte deutlich zu erweitern und auszudifferenzieren: Der Zugriff auf und Zugang zum Fahrzeug verändern sich, indem nicht mehr der Nutzer zum Fahrzeug, sondern das Fahrzeug zum Nutzer kommt; die Fahrzeuge selbst werden für einen erweiterten Personenkreis wie z. B. mobilitätseingeschränkte Menschen nutzbar; neue Formen von öffentlichem Verkehr sind denkbar, auch im Sinne einer weiteren Aufweichung der Grenzen zwischen Individualverkehr und öffentlichem Verkehr.

Der vorliegende Beitrag hat das Ziel, diese Optionen und die damit verbundenen Erwartungen vorzustellen. Dabei konzentrieren sich die Ausführungen und die Diskussion auf das Carsharing. In einem ersten Schritt wird der derzeitige Stand von Angebot und Nutzung der sogenannten „Neuen Mobilitätskonzepte“, bei denen das Carsharing den Kern bildet, dargestellt. Den Hauptteil des Beitrags bildet die Diskussion zu den Chancen und Herausforderungen, die sich durch die Einführung von autonom fahrenden Fahrzeugen in Carsharing-Flotten ergeben würden. Derzeit gibt es eine Reihe von Anzeichen dafür, dass Spontaneität und Flexibilität eine besonders hohe Bedeutung für die Nutzung der neuen Mobilitätskonzepte haben könnten (vgl. [11, 13]). Genau an dieser Stelle, nämlich der Erhöhung von Spontaneität und Flexibilität, könnten neue Mobilitätskonzepte mit autonomen Fahrzeugen ansetzen. Entsprechende Überlegungen sind bei Betreibern von flexiblen Carsharing-Flotten bereits im Entstehen [4].

\subsection{Carsharing: „Kernapplikation“ neuer Mobilitätskonzepte}

Carsharing gibt es in Deutschland und zahlreichen anderen Ländern etwa seit den 1980erJahren. Unter Carsharing wird hier der Betrieb einer Pkw-Flotte verstanden, die entweder stationsbasiert oder Punkt-zu-Punkt verfügbar ist. Jeder Inhaber und jede Inhaberin einer gültigen Fahrerlaubnis kann sich als Mitglied einer Carsharing-Organisation - in der Regel bei gleichzeitiger Entrichtung einer Anmeldegebühr - registrieren lassen und erhält damit Zugang zu den Fahrzeugen. Die wesentlichen Variationen des Carsharing ergeben sich aus den räumlichen und zeitlichen Zugriffsbedingungen auf die Fahrzeuge sowie aus den Geschäftsmodellen; Abb. 9.1 fasst die Ausprägung der verschiedenen Konzepte in ihren Grundzügen zusammen. 


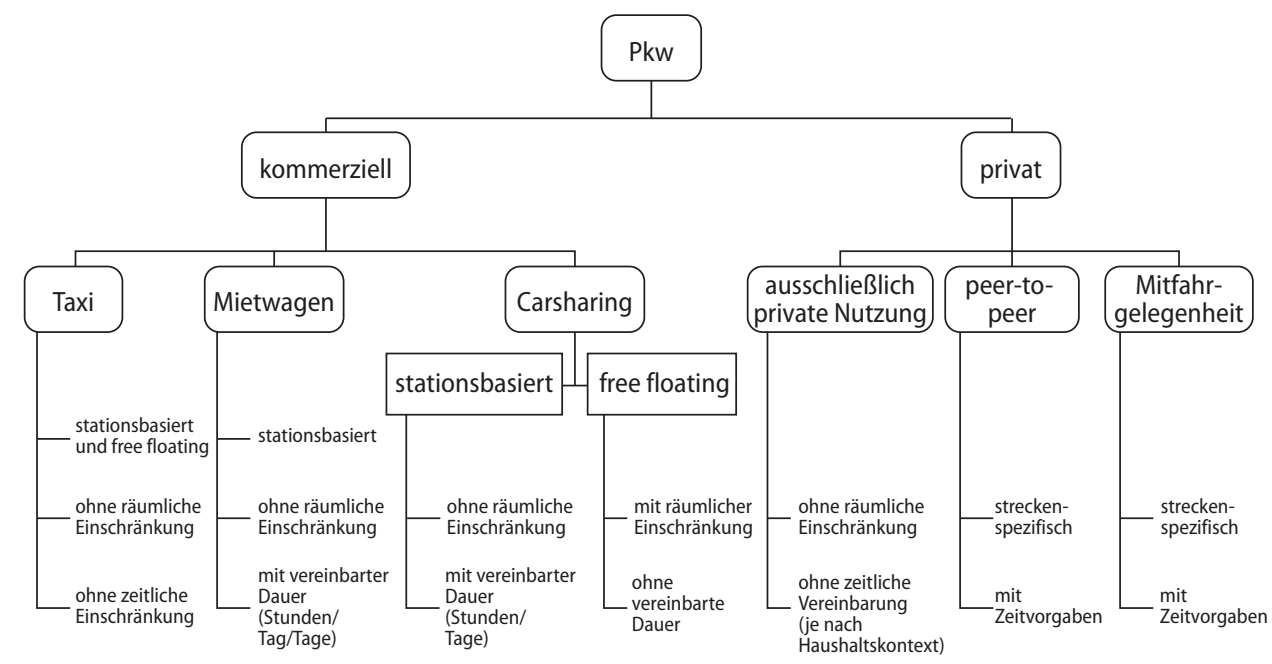

Abb. 9.1 Systematik zur Pkw-Nutzung im Spannungsfeld zwischen privatem und kommerziellem Einsatz

\subsubsection{Stationsbasiertes Carsharing}

Die klassische Form des Carsharing ist das stationsbasierte Carsharing, d.h., an einem „Sammelpunkt“" werden die Fahrzeuge bereitgestellt. Der Nutzer muss das Fahrzeug dort abholen und auch wieder dorthin zurückbringen. Die Dauer der Nutzung wird vorab vereinbart. Im Unterschied zum klassischen Mietwagen können die stationsbasierten Carsharing-Fahrzeuge nicht nur tage-, sondern auch stundenweise entliehen werden; allerdings gibt es zunehmend auch bei Mietwagen-Anbietern die Möglichkeit zur stundenweisen Anmietung eines Fahrzeugs. Die Nutzer zahlen eine jährliche Grundgebühr; für die Fahrzeugnutzung wird ein aus Mietdauer und gefahrener Entfernung abgeleitetes nutzungsabhängiges Entgelt erhoben, das je nach Organisation und Anbieter variiert und auch der Nachfrage entsprechend an Tageszeit und Wochentag angepasst sein kann. Ein Geschäftsgebiet ist nicht festgelegt. In der Anfangszeit fand die Reservierung der Fahrzeuge überwiegend telefonisch statt, heute erfolgen Buchungen auch über die Anbieter-Website oder über Anwendungen auf mobilen Endgeräten.

Im Jahr 2014 ist stationsbasiertes Carsharing an circa 3900 Stationen in 380 Städten und Gemeinden Deutschlands verfügbar [5], [6]. Dazu gehört auch eine ganze Reihe mittlerer und kleinerer Städte; in Städten über 100.000 Einwohnern wird schon eine sehr gute Abdeckung erreicht, dagegen gibt es in Deutschland in weniger als 5 Prozent der Kommunen mit einer Einwohnerzahl von unter 50.000 ein Carsharing-Angebot [7]. Zur Bedienung der 3900 Stationen steht eine Flotte mit 7700 Fahrzeugen zur Verfügung, die von etwa 150 Carsharing-Anbietern bereitgestellt wird. Marktführer in Deutschland ist die Deutsche Bahn (Flinkster), der etwa 55 Prozent der stationsbasierten Flotte gehört [8, 9]. Weltweiter 
Marktführer im klassischen Carsharing mit einer Flotte von rund 10.000 Fahrzeugen in den USA sowie Kanada, Großbritannien, Spanien und Österreich ist das im Jahr 2000 gegründete amerikanische Unternehmen Zipcar, das inzwischen zur AVIS Budget Gruppe gehört (Stand August 2014).

\subsubsection{Flexibles (One Way) Carsharing}

In den vergangenen Jahren sind neue Formen von Carsharing entstanden. Als neuen Typ des gewerblichen Carsharing gibt es inzwischen vor allem in Deutschland, aber auch in Großbritannien und den USA, das sogenannte ,flexible“ Carsharing, dessen Flexibilität hauptsächlich darin besteht, dass keine vorherige Vereinbarung mit dem Betreiber über Zeitpunkt und Dauer der Nutzung getroffen und das Fahrzeug nicht an einem bestimmten Ort abgeholt und dorthin zurückgebracht werden muss. Vielmehr nehmen die Nutzer das Fahrzeug dort auf, wo sie es antreffen, und geben es an beliebiger Stelle innerhalb des vom Betreiber definierten Geschäftsgebietes ab. Die Informationen über den Standort ausleihbarer Fahrzeuge erhalten die Nutzer aus dem Internet oder über eine Smartphone-App; grundsätzlich kann das Fahrzeug auch en passant ausgeliehen werden, d.h., man leiht ein an der Straße geparktes, nicht reserviertes Fahrzeug aus. Auch hier ist die Registrierung beim Betreiber der Flotte Voraussetzung für die Nutzung des Fahrzeugs, die per Chipkarte oder neuerdings direkt über das Smartphone initiiert wird.

Das weltweit führende Unternehmen im flexiblen Carsharing ist Car2Go mit mehr als 10.000 Fahrzeugen in 27 Städten in Europa und Nordamerika (Stand August 2014). In Deutschland verfügen die Anbieter des flexiblen Carsharing über eine Flotte von insgesamt etwa 6250 Fahrzeugen [5], die allerdings fast ausschließlich in großen Städten mit mehr als 500.000 Einwohnern, so beispielsweise in Berlin, Hamburg oder München, angeboten werden. Dabei umfassen die Geschäftsgebiete nicht das gesamte städtische Territorium, sondern sind auf Teile davon beschränkt, hauptsächlich auf das Innenstadtgebiet sowie angrenzende Gebiete und „Inselgebiete“, in denen eine hohe Nutzungsfrequenz gegeben ist. Neben einer einmaligen Anmeldegebühr fallen keine weiteren regelmäßigen Kosten an. Die Nutzung der Fahrzeuge wird über einen zeitabhängigen Nutzungstarif, meist pro Minute, berechnet. Kosten für den Treibstoff sind wie beim stationsbasierten Carsharing in den Tarifen enthalten; darüber hinaus enthält die Nutzungsgebühr die Parkkosten, die üblicherweise als Pauschale direkt zwischen Anbieter und Kommune vereinbart sind.

Flexibles Carsharing startete als Pilotprojekt von Car2Go, einem Unternehmen des Daimler-Konzerns, im Jahr 2009 in Ulm (Baden-Württemberg, Deutschland). Weitere relevante Anbieter sind inzwischen das seit 2011 aktive DriveNow, ein Unternehmen von BMW und der Sixt-Autovermietung, sowie der mit rein batterie-elektrisch betriebenen Fahrzeugen ausgestattete Dienst Multicity, einer Kooperation von Citroën mit der Deutschen Bahn. Gleichzeitig versuchen sich zunehmend neue Anbieter im Markt, wie beispielsweise Quicar, das der Volkswagen-Gruppe angehört, oder Spotcar von Opel, das im Juni 2014 in Berlin gestartet ist. 


\subsubsection{Peer-to-Peer Carsharing}

Das Peer-to-Peer Carsharing, also das Verleihen des privaten Pkw zwischen Privatpersonen, ist eben erst dabei, sich als dritte Systemform des Carsharing über Vermittlungsplattformen im Internet zu entwickeln. Genaue Nutzerzahlen liegen hierzu noch nicht vor. Die Buchungen werden beim Peer-to-Peer Carsharing über eine Internetplattform abgewickelt. Es gibt für das Abholen und Zurückbringen keine Station, vielmehr wird das Fahrzeug an einem individuell vereinbarten Standort abgeholt bzw. dorthin zurückgebracht. Der Blick auf eine Webplattform wie www.autonetzer.de zeigt, dass diese Form des Carsharing sich keineswegs nur auf die großen Städte beschränkt, sondern auch in kleineren Städten und Kommunen zu finden ist. Darin scheint sich die Vermutung von Hampshire und Gaites ([10], S.14) zu bestätigen, dass es sich bei Peer-to-Peer Carsharing - zumindest derzeit noch im Gegensatz zum kommerziellen Carsharing - um eine skalierbare Form des Carsharing handelt. Dabei ist die Flottenzusammensetzung ausgesprochen dynamisch, da es sich bei der Bereitschaft zum Verleihen des Pkw nicht um eine grundsätzliche Verhaltensweise seitens der Anbieter zu handeln scheint. Vielmehr legen die Ergebnisse einer Untersuchung in Berlin die Annahme nahe, dass die Pkw-Besitzer ihre Fahrzeuge nur in bestimmten Zeiten zur Verfügung stellen, beispielsweise dann, wenn die eigene Nutzung des Pkw aus persönlichen Umständen heraus gering ist [11].

\subsection{Nutzer und Nutzung der neuen Mobilitätskonzepte}

Die Zielsetzungen, die mit Carsharing verbunden werden, variieren je nach Perspektive der beteiligten Akteure. Seitens der Politik, die in Deutschland hauptsächlich auf kommunaler Ebene in die Bereitstellung der notwendigen Rahmenbedingungen für die Implementierung von Carsharing eingebunden ist, sind die Reduzierung des Verkehrsaufkommens mit dem Pkw (da Carsharing-Nutzer mit der Dauer der Carsharing-Nutzung auch den ÖPV-Anteil innerhalb ihres Modal Split erhöhen) und damit verbunden eine Verringerung der Emissionen von Luftschadstoffen und $\mathrm{CO}_{2}$ sowie die Minderung des Flächenanspruches des ruhenden Pkw-Verkehrs wesentliche Motive bei der Unterstützung von Carsharing-Initiativen (vgl. Kap. 19). Kommerzielle Betreiber von Carsharing, wie Automobilhersteller (in der Regel in Kooperation mit Autovermietern) oder Mobilitätsdienstleister wie die Deutsche Bahn, verfolgen mit Carsharing produktbezogene Strategien, so u. a. die Erweiterung ihres Leistungsangebotes durch eine (zusätzliche) Mobilitätsdienstleistung oder die Bereitstellung von attraktiven Fahrzeugen ihrer Marke und damit die Erzeugung von Markenbindung. Andere Motive für den Betrieb einer Carsharing-Flotte sind dezidiert ökologische Zielsetzungen, die wesentlich die Entstehung von Carsharing angetrieben haben und die insbesondere von den als Verein oder als Interessengemeinschaft organisierten Gruppen weiterhin aufrechterhalten werden [12], [13]. 


\subsubsection{Nutzer und Nutzungsvoraussetzungen}

Die Carsharing-Nutzer des Jahres 2014 bilden eine spezifische Gruppe sowohl hinsichtlich ihrer Zusammensetzung als auch hinsichtlich ihres Mobilitätsverhaltens. Deutlich höher verglichen mit dem Bevölkerungsdurchschnitt sind: der Anteil an Personen unter 40 Jahren, der Anteil an Männern, der Anteil an Personen mit hohem formalem Bildungsstand (Abitur, Fachhochschulreife, Studium) und das Haushaltseinkommen. Diese Differenz zum Bevölkerungsdurchschnitt ist beim flexiblen Carsharing noch stärker ausgebildet als beim stationsbasierten [8], [9]. Beide Typen von Carsharing - stationsbasiert und flexibel werden kombiniert mit einer weit überdurchschnittlichen Nutzung des öffentlichen Verkehrs. So zeigen Untersuchungen zur Nutzung von Carsharing aus dem Jahr 2014, dass 52 Prozent der Flinkster-Kunden in Berlin und 44 Prozent der Münchner Flinkster-Kunden eine ÖPV-Zeitkarte besitzen. Bei den flexiblen Systemen DriveNow und Car2Go verfügt ebenfalls ein hoher Anteil über ein ÖPV-Abo: Bei DriveNow in Berlin und München sind es 43 Prozent bzw. 38 Prozent ([14], S.12); bei Car2Go haben 40 Prozent der Nutzer in Stuttgart und 50 Prozent der Nutzer in Köln eine Dauerkarte für den öffentlichen Verkehr ([15], S.13); im Bundesdurchschnitt liegt der Anteil der Bevölkerung, die in Kernstädten wohnt und eine Zeitkarte für den öffentlichen Verkehr besitzt, bei 33 Prozent ([1], eigene Auswertung; die amtliche Raumbeobachtung in Deutschland zählt zur Kategorie „Kernstadt" alle kreisfreien Städte mit mehr als 100.000 Einwohnern).

Ein gut ausgebauter öffentlicher Verkehr oder der Besitz eines eigenen Fahrzeugs scheinen derzeit wesentliche Voraussetzungen für die Nutzung von Carsharing zu sein. In der Variante des flexiblen Carsharing ist nur so eine One-Way-Nutzung möglich. Müsste der Nutzer diejenigen Wege, die nicht mit einem Carsharing-Fahrzeug zurückgelegt werden, selbst anderweitig organisieren, wäre der Aufwand höchstwahrscheinlich hoch und die Attraktivität eines Systems, das die Nutzung einzelner Strecken erlaubt, würde deutlich sinken. Auch im Fall von stationsbasiertem Carsharing bildet der öffentliche Verkehr häufig das hauptsächliche Verkehrsmittel für die Nutzer; vielfach stellt das Carsharing-Fahrzeug auch die bedarfsweise Ergänzung Pkw-besitzender Haushalte um ein weiteres Fahrzeug dar [16]. Um diesen Bedarf zu bedienen und im Sinne einer Symbiose auszunutzen, existieren seit Langem schon Kooperationen zwischen den Anbietern von stationsbasiertem Carsharing und öffentlichen Verkehrsdienstleistungen; eine vergleichbare Zusammenarbeit findet sich auch beim neuen flexiblen Carsharing [17].

\subsubsection{Der Carsharer - der „neue Mensch“ in einer Sharing Economy?}

Unter dem Schlagwort vom „Nutzen statt besitzen” wird Carsharing immer wieder als Beispiel für den Wandel der Besitz-Ökonomie hin zu einer Ökonomie des Teilens, einer Sharing Economy, herangezogen [18, 19]. Möglicherweise resultiert dies aus der besonderen Offensichtlichkeit des Carsharing als eines Tuns, das im öffentlichen Raum stattfindet. Möglicherweise steht dahinter aber auch das Staunen darüber, dass ein Gegenstand, der 
nach wie vor zum Statussymbol taugt, im Verleihverfahren von mehreren, beliebigen Personen genutzt wird.

Tatsächlich reiht sich das Carsharing ein in eine ganze Reihe von Entwicklungen, bei denen „Güter“ auf Leihbasis ,geteilt" werden, die bislang als nicht verleihbar galten: die selbst genutzte Wohnung, der Schrebergarten, das Auto. Ökonomen begründen die Verleihbarkeit (oder Nicht-Verleihbarkeit) eines Gutes mit der Differenz zwischen den entstehenden Transaktionskosten und den Einnahmen, die über den Verleih generiert werden können; entsteht eine positive Differenz, ist der Verleih begründet, und er ist umso interessanter, je höher die Differenz ausfällt [20]. Das Verleihen wird für den Einzelnen jedoch nur dann sinnvoll, wenn er über ein Produkt verfügt, dessen Kapazität er unvollständig nutzt - deshalb der Verleih des eigenen Autos in Standzeiten, der Verleih der Wohnung, solange man selbst im Urlaub ist, der Verleih des privaten Parkplatzes vor dem Haus, während man tagsüber bei der Arbeit ist.

Die Auseinandersetzung mit den Wirkungen eines geteilten Nutzens stammt - zumindest in Deutschland - im Wesentlichen aus der Diskussion um die nachhaltige Nutzung von Ressourcen [21, 22, 23]. Dabei zeigt sich das Sharing auf aggregierter Ebene, d.h. auf regionaler, nationaler oder auch supra-nationaler Ebene, als eine Möglichkeit zur Ressourceneinsparung. Auf individueller Ebene dagegen bedeutet Sharing nicht weniger Konsum, sondern die Möglichkeit zu gleichbleibend hohem oder auch mehr Konsum. Als Beispiel hierfür kann in der Tat das Carsharing angesehen werden, bei dem den Mitgliedern der jeweiligen Organisation eine Vielfalt an Fahrzeugen oder doch mehrere Fahrzeugtypen zur Verfügung gestellt werden. Diese Fahrzeuge bieten eine Breite an Konsumgütern des Typs Fahrzeug, die die Möglichkeiten von Privathaushalten mehrheitlich übersteigen würden, wenn sie diese Bandbreite an Fahrzeugen selbst besitzen wollten. Auf aggregierter Ebene werden weniger Fahrzeuge benötigt (der Bundesverband Carsharing (BCS) gibt an, dass sich - rein rechnerisch - im stationären Carsharing 42 Personen, im flexiblen Carsharing 70 Personen ein Fahrzeug teilen [5]); auf individueller Ebene bleibt damit eine hohe Mobilität gewährleistet.

In den Anfangsjahren war das Carsharing oft verbunden mit einer Einstellung, bei der dem Autofahren als solchem kein „Handlungsnutzen“ zugesprochen wurde - sprich: AutoNutzer mit einer entsprechenden Einstellung empfanden keinen Spaß am Autofahren (vgl. [21], S.92). Vielmehr war die Nutzung eher aus einer Einstellung heraus motiviert, die der wachsenden Motorisierung der privaten Haushalte eine wesentliche Mitverantwortung an der Verschlechterung der Umweltbedingungen gab, der die Carsharing-Mitglieder entgegenwirken wollten [12]. Dies hat sich möglicherweise grundlegend geändert. So hat sich das Produkt Carsharing - sowohl in der flexiblen und zunehmend auch in der stationsbasierten Version - zu einem kommerziellen Produkt entwickelt. Darüber hinaus belegen Untersuchungen zur Nutzung von flexiblem Carsharing, dass Aspekte wie „Aufmerksamkeit, erlebte Sympathie, Spaß und Begeisterung" ganz wesentliche Motive sind (vgl. [24] S.21: Zustimmungsraten zu entsprechenden Aussagen zwischen 38 und 86 Prozent). Damit übernimmt das Carsharing-Fahrzeug bzw. die Carsharing-Nutzung emotionale und psychosoziale Funktionen, die dem Auto bislang nur in Form von Eigentum zugesprochen wurden 
[22]. Bardhi und Eckhardt verstehen das „Teilen“ (von den Autorinnen zutreffender als access-based consumption bezeichnet) als charakteristisches Merkmal einer liquid society, in der feste Bezugssysteme, wie sie auch durch Eigentum entstehen, zunehmend brüchig werden [24].

\subsection{Digitalisierung der Alltagswelt als Grundvoraussetzung für neue Mobilitätskonzepte}

Die Entwicklung und Erweiterung des Carsharing durch neue Konzepte wie das flexible Carsharing oder auch das Peer-to-Peer Carsharing ist ohne die Verfügbarkeit von Geräten, die einen mobilen Zugriff auf das Internet und Kommunikationsanwendungen wie beispielsweise Apps erlauben, nicht denkbar. Zwar präsentieren alle Anbieter ihr jeweiliges Carsharing-Produkt auch umfangreich im (stationären) Internet; hierbei geht es aber eher um die Information für die (potenziellen) Kunden als um die unmittelbare Nutzung des Dienstes. Für den Zugang zu den Fahrzeugen spielen vor allem die mobilen Anwendungen eine wesentliche Rolle. Sie ermöglichen zunächst die Verortung von Nutzer und Fahrzeug(en) in Echtzeit; damit erhält der Nutzer die notwendige Grundlage, um sich über die Verfügbarkeit eines Fahrzeuges zu informieren und zu entscheiden, ob er den Weg zum Fahrzeug (für den er derzeit je nach Anbieter zwischen 15 und 30 Minuten zur Verfügung hat) zurücklegen will und kann. Im zweiten Schritt ermöglicht die mobile Anwendung die Reservierung des ausgewählten Fahrzeugs und bietet eine Navigation zu diesem Fahrzeug an. Auch das Öffnen des Fahrzeugs erfolgt bei einzelnen Anbietern schon heute über die mobile Anwendung.

Dabei ist die Einsatzmöglichkeit entsprechender Technologien entscheidend davon abhängig, dass die Nutzer ihrerseits über die technische Ausstattung verfügen, mit der sie auf das mobil und digital vermittelte Fahrzeugangebot zugreifen können. Tatsächlich hat der private Besitz von Smartphones in den vergangenen Jahren erheblich zugenommen. Während im Jahr 2009 erst knapp 6,5 Millionen Personen in Deutschland ein Smartphone besaßen, sind es heute über 40 Millionen, also fast jede(r) Zweite. Für das Jahr 2014 wird erwartet, dass 97 Prozent der verkauften Handys Smartphones sein werden; gerechnet wird mit dem Verkauf von fast 30 Millionen Geräten [25, 26].

Gleichzeitig ist davon auszugehen, dass die Fertigkeiten im Umgang mit Hard- und Software in allen Gruppen der Bevölkerung zunehmen. So stellt beispielsweise die ARDZDF-Onlinestudie 2013 fest, dass die Zahl der Internetnutzer weiterhin expandiert [27]: Im Jahr 2013 waren in Deutschland 54,2 Millionen Personen ab 14 Jahren zumindest gelegentlich online; das sind 77,2 Prozent der Bevölkerung und damit 800.000 Menschen mehr als noch im Vorjahr. Verursacher dieses Wachstums ist ausschließlich die Bevölkerungsgruppe der über 50-Jährigen. Gleichzeitig nimmt die Nutzungsdauer zu: 2013 war der deutsche Internetnutzer im Schnitt 169 Minuten am Tag online, das sind 36 Minuten mehr als im Vorjahr. Einen beträchtlichen Anteil daran hat die Unterwegs-Nutzung des Internets, die im Jahr 2012 erst von 23 Prozent der Nutzer praktiziert wurde, im Jahr 2013 aber bereits von 
41 Prozent. Apps werden von fast der Hälfte der Online-Nutzer (44 Prozent) auf unterschiedlichen Endgeräten genutzt [28].

Im verkehrlichen Kontext besonders beachtenswert ist die durch digitale Anwendungen gegebene und durch mobile Endgeräte noch deutlich verstärkte Möglichkeit zur Reduzierung von Planungshorizonten. Spontaneität in der Gestaltung der individuellen Mobilität ist damit eine besonders wichtige Konnotation zum meistgebrauchten Begriff der „Flexibilität" als spezifischer Eigenschaft der neuen Mobilitätskonzepte. Entsprechend war das mit der höchsten Zustimmung versehene Statement in einer im Jahr 2014 durchgeführten Befragung von Car2Go-Nutzern: „Ich finde an Car2Go attraktiv, dass ich spontan ein Auto nutzen kann, auch wenn ich ohne Auto unterwegs bin“-98 Prozent der Befragten stimmten dieser Aussage zu (72 Prozent ,trifft genau zu“, 26 Prozent ,trifft eher zu“) (vgl. [15], S.20). Damit wird auch der schnelle Erfolg des flexiblen Carsharing nachvollziehbar, das von der (langen) Vorausplanung, wie sie beim konventionellen stationsbasierten Carsharing notwendig war, entbindet und zumindest mittelfristig auch dem privaten Pkw den bislang geltenden Vorteil der permanenten Verfügbarkeit nehmen könnte (vgl. dazu auch [22], S.64).

Insgesamt bedeutet dies, dass wohl kaum mit größeren Zugangshürden seitens der (potenziellen) Nutzer zu rechnen ist, sofern künftige Mobilitätskonzepte an Praktiken anknüpfen, wie sie bereits heute geübt werden (,geübt“ im wahrsten Sinne des Wortes; s. analog dazu die Einübung der Interaktion zwischen Mensch und Computer bei der Fahrzeugnavigation Kap.3). Wenig wahrscheinlich, wenngleich immer wieder auch von wissenschaftlicher Seite ins Feld geführt, erscheint allerdings die Umstellung der kompletten Fahrzeugflotte auf Fahrzeuge, die als Sharing-Fahrzeuge oder als Fahrzeuge von Betreibern öffentlicher Verkehre unterwegs sind. Es gibt derzeit keinerlei Anzeichen dafür, dass der private Pkw an Attraktivität verliert; laut Kraftfahrtbundesamt (KBA) gab es zum 1. Januar 2014 einen neuen Höchststand beim Fahrzeugbestand in Deutschland. Der PkwBestand war zwischen 2013 und 2014 um rund 500.000 Fahrzeuge angewachsen und fügt sich damit in einen längerfristigen Wachstumstrend ein [29].

\subsection{Weiterentwicklung der neuen Mobilitätskonzepte durch Automatisierung des Carsharing?}

Für die Weiterentwicklung der vorhandenen Carsharing-Konzepte durch autonome Fahrzeuge gibt es eine Reihe von Varianten, die sich analog zu den in Kap. 2 abgebildeten Anwendungsfällen - im Folgenden „Use-Cases“ - verhalten. Diese Varianten zielen auf unterschiedliche Nutzerbedürfnisse ab, die so auch heute schon adressiert werden, allerdings über den Selbstfahrer. Im Folgenden soll entlang der Use-Cases „Autonomes ValetParken“, „Vollautomat mit Verfügbarkeitsfahrer“ und „Vehicle-on-Demand“ diskutiert werden, welche Veränderungen Carsharing durch die Einflottung autonomer Fahrzeuge erfahren würde und welche Wirkungen für den Nutzer sich davon erwarten lassen. Dabei wird auch der Wettbewerb mit derzeit bestehenden Transportangeboten anzusprechen sein. 
Bei allen Use-Cases wird der höchste Automatisierungsgrad unterstellt, das ist nach der Nomenklatur der BASt der Automatisierungsgrad ,vollautomatisiert“ [30]. Der Unterschied zwischen den Use-Cases liegt in den dafür definierten Anwendungsfällen: Während es beim autonomen Valet-Parken ausschließlich um das Abstellen bzw. Holen des Fahrzeugs geht, bezieht sich der Vollautomat mit Verfügbarkeitsfahrer auf grundsätzlich jeden im Straßenverkehr denkbaren Anwendungsfall, wenngleich der Schwerpunkt auf Situationen mit vergleichsweise einfachen Mischverkehren liegt, d.h. auf Verkehren, wie sie beispielsweise auf Autobahnen gegeben sind, wo jedoch auch hohe Geschwindigkeiten die Regel sind. Beim Vollautomat mit Verfügbarkeitsfahrer muss der Fahrer die Fahraufgabe dann zeit- und streckenabschnittsweise übernehmen, wenn er Streckenabschnitte durchqueren möchte, die permanent oder temporär von der Freigabe für autonomes Fahren ausgenommen sind. Das Vehicle-on-Demand ist ebenfalls in der Lage, jeden im Straßenverkehr denkbaren Anwendungsfall zu bewältigen, und zwar auch Situationen mit Mischverkehren. Durch den Verzicht auf den sogenannten Fahrerarbeitsplatz, also den Sitzplatz, von dem aus der Fahrer oder die Fahrerin die Fahraufgabe ausführt, sind die Nutzungsmöglichkeiten des Fahrzeuginnenraums deutlich vielfältiger als beim Valet-Parken oder beim Vollautomaten mit Verfügbarkeitsfahrer.

\subsubsection{Autonomes Valet-Parken im Carsharing}

Der Use-Case des autonomen Valet-Parkens geht davon aus, dass das Fahrzeug sich selbstständig vom Parkplatz zum Nutzer bzw. vom Nutzer zum Parkplatz bewegen kann, auch unter Verwendung einer öffentlichen Straße.

Der Einsatz von autonomem Valet-Parken im Carsharing würde zunächst bedeuten, dass der Aufwand des Nutzers für die Beschaffung des Fahrzeugs und für die an die Nutzung sich anschließende Parkierung deutlich sinken würde. Vielmehr entstünde nun aus der Sicht des Nutzers ein Tür-zu-Tür-Service - vergleichbar mit einer Taxifahrt, bei der allerdings der Nutzer die Fahraufgabe bei der eigentlichen Fahrt selbst übernimmt. Die für Mobilität aufzuwendende Zeit würde in jedem Fall reduziert, da Zu- und Abgangszeiten entfallen.

Um die Attraktivität des Angebots zu steigern, wären - unabhängig von der Automatisierung - Erweiterungen denkbar in Hinblick auf Wahlmöglichkeiten bei der Ausstattung des Fahrzeugs, der Anzahl der Sitzplätze und der Transportkapazität oder auch Internetverfügbarkeit und Multi-Media-Angebote. Wie stark differenziert die Wahlmöglichkeiten sein könnten, wäre - wie auch beim heutigen Carsharing - abhängig von der Flottengröße, der Anzahl an (potenziellen) Kunden pro Fahrzeug und der Größe des Geschäftsgebietes, aber auch von der Zahlungsbereitschaft der Kunden für unterschiedliche Ausstattungsvarianten. Bei einer großen Flotte wäre es auch denkbar, die Fahrzeugdifferenzierung mit einer preislichen Differenzierung zu hinterlegen, vergleichbar der gängigen Praxis beim stationsbasierten Carsharing oder auch der klassischen Autovermietung. Dem stehen im flexiblen Carsharing derzeit Geschäftsmodelle gegenüber, die für die Nutzer möglicherweise da- 
durch einen zusätzlichen (An-)Reiz bieten, dass sie aus der bestehenden Flotte eine große Bandbreite an Fahrzeugen zu den gleichen Kosten bekommen können.

Mit dem autonomen Valet-Parken wird das Bereitstellen von Fahrzeugen an einer Station grundsätzlich überflüssig; das gesamte Carsharing-Angebot ließe sich damit auf flexibles Carsharing umstellen, zumindest würde die Abholung und Rückführung des Fahrzeugs durch den Nutzer überflüssig werden. Zwar werden die Betreiber nicht auf Stationen oder Fahrzeugdepots verzichten, um eine gewisse Zentralisierung und damit Effizienz bei der Wartung der Fahrzeuge zu erzielen. Diese Stationen müssten dann aber nicht mehr in möglichst großer räumlicher Nähe zum Kunden aufgebaut werden, sondern könnten Flächen nutzen, für die geringere Kosten entstehen. Die Distanz zu den Orten der Nutzung ist dabei nicht beliebig, da für den Weg zum Kunden nur ein vergleichsweise enges Zeitfenster zur Verfügung stehen dürfte. Außerdem ist die Anfahrt zum Kunden mit dem Verbrauch von Ressourcen verbunden und stellt gleichzeitig - sofern keine andere Nutzung auf diesem Weg möglich ist - ,tote“ Zeit im Fahrzeuggebrauch dar.

Alternativ oder ergänzend dazu könnten mehrere kleinere Sammelpunkte mit einigen wenigen Fahrzeugen relativ dicht verteilt über das Geschäftsgebiet entstehen. Damit würde der Zeitaufwand zwischen Fahrzeugbestellung und Abholung des Nutzers gering gehalten; möglich wäre dann auch eine Mischform der Fahrzeuginanspruchnahme mit wahlweise autonomer Zufahrt oder persönlicher Abholung. Damit ließen sich die aktuell noch sehr unterschiedlichen Zugangszeiten zu den Fahrzeugen, je nach kleinräumiger Dichte der Fahrzeugverfügbarkeit, deutlich nivellieren. Momentan liegen die Zugangszeiten, berechnet aus dem Unterschied zwischen den gemessenen Werten „Buchungszeitpunkt/Reservierung“ und „Fahrtdauer“, zwischen 1-16 Minuten (Beispielsfall DriveNow mit maximaler Reservierungszeit von 15 Minuten; Quelle: Projekt WiMobil, gefördert vom Bundesumweltministerium BMUB). Die Unterschiede in der durchschnittlichen Dauer der Zugangszeit hängen unmittelbar mit den unterschiedlichen Fahrtzwecken zusammen.

\subsubsection{Carsharing unter Einsatz von „Vollautomaten mit Verfügbarkeitsfahrer"}

Der Use-Case des Vollautomaten mit Verfügbarkeitsfahrer geht davon aus, dass sich das Fahrzeug grundsätzlich selbstständig auf öffentlichen Straßen bewegen kann, dass aber die Fahraufgabe individuell und zeitweise vom Fahrer oder der Fahrerin wieder übernommen wird.

Aus einer Carsharing-Perspektive bleiben die mit dem „Vollautomaten“ möglichen Veränderungen und Erweiterungen deutlich hinter dem autonomen Valet-Parken zurück, zumindest dann, wenn der Verfügbarkeitsfahrer als Fahrzeuginsasse vorausgesetzt wird. Der einzige Unterschied, der sich in diesem Use-Case zum heutigen Carsharing ergeben würde, wäre die Möglichkeit, während der Fahrt die Fahraufgabe an das Fahrzeug abzugeben, wenn der Fahrer dies wünscht. Manche Streckenabschnitte können für die autonome 
Fahrfunktion allerdings auch dauerhaft nicht zugelassen sein, ,z.B. Strecken mit einer hohen Fußgängerüberquerfrequenz" (s. Kap. 2). Dies wären allerdings vor allem Bereiche im städtischen Umfeld, das - zumindest zum jetzigen Zeitpunkt - das Hauptnutzungsgebiet für das flexible Carsharing darstellt.

Die Erweiterung von Carsharing mit Fahrzeugen im Sinne des Vollautomaten mit Verfügbarkeitsfahrer würde demnach eher auf Strecken am Rand oder außerhalb von Siedlungen einen nachvollziehbaren Nutzen erzeugen. Allerdings lässt sich daraus wohl kaum ein Geschäftsmodell für ein „Carsharing im ländlichen Raum“ ableiten, da durch die Notwendigkeit des Verfügbarkeitsfahrers eine Bedienung des Geschäftsgebietes über ein Fahrzeug, das die Zufahrt und Wegfahrt selbstständig übernimmt, nicht möglich ist.

Insgesamt ist der Einsatz des Vollautomaten mit Verfügbarkeitsfahrer den Möglichkeiten, die sich durch autonomes Valet-Parken ergeben würden, deutlich unterlegen.

\subsubsection{Das Carsharing-Fahrzeug als Vehicle-on-Demand}

Der Use-Case des Vehicle-on-Demand geht davon aus, dass sich das Fahrzeug selbstständig auf allen öffentlichen Straßen bewegt. Ein Fahrer ist - auch als Rückfallebene - nicht notwendig. Damit wird auch kein Fahrerarbeitsplatz mehr benötigt, was neue Gestaltungsmöglichkeiten für den Innenraum des Fahrzeugs eröffnet.

Als Carsharing-Fahrzeug verfügt das Vehicle-on-Demand zunächst über die gleichen Vorteile wie das Carsharing-Fahrzeug mit der Funktion des Valet-Parkens, allerdings wird aus dem Fahrzeug zusätzlich eine Art ,,auf der Straße rollendes Abteil““. Damit sind in diesem Fahrzeug ganz unterschiedliche Beschäftigungen möglich, wie z. B. das Lesen, Spielen, Telefonieren, Arbeiten oder Dösen, und der Nutzer kann dazu jeden beliebigen Platz im Fahrzeug einnehmen. Unterstellt man Nutzungsdauern, wie sie heute im flexiblen Carsharing üblich sind, erscheint es jedoch offen, ob der Zusatznutzen, der durch den Wegfall der Fahraufgabe entsteht, tatsächlich hoch bewertet wird - so liegt beispielsweise die durchschnittliche Nutzungsdauer eines Fahrzeugs in einer Flotte wie der von DriveNow im Jahr 2014 bei rund einer halben Stunde [31]. Geht man davon aus, dass autonom fahrende Carsharing-Fahrzeuge für alle im Laufe eines Tages anfallenden Arbeitswege genutzt werden, würde diese Zeitspanne in den größeren Städten nach heutigem Stand durchschnittlich 54 Minuten ausmachen, für Menschen, die im Umland der größeren Städte oder im ländlichen Raum wohnen, beträgt der Gesamtaufwand für Arbeitswege während eines Werktages derzeit 50 bzw. 49 Minuten (Datenquelle: MiD 2008, [1]). Dabei gibt es nahezu keinen Unterschied hinsichtlich der Zeitaufwände, die in den verschiedenen Raumtypen für Arbeitswege mit dem Auto aufgewendet werden (s. Abb. 9.2).

Die Fahrt in einem Vehicle-on-Demand im Carsharing würde einer Fahrt mit dem Taxi ähneln, und da das Fahrzeug einem breiten Nutzerkreis zur Verfügung steht, höchstwahrscheinlich auch Taxifahrten ersetzen. Dies wird jedoch in Abhängigkeit von den Kosten zu sehen sein, die für eine Fahrt mit einem autonomen Carsharing-Fahrzeug im Vergleich zum Taxi entstehen. 


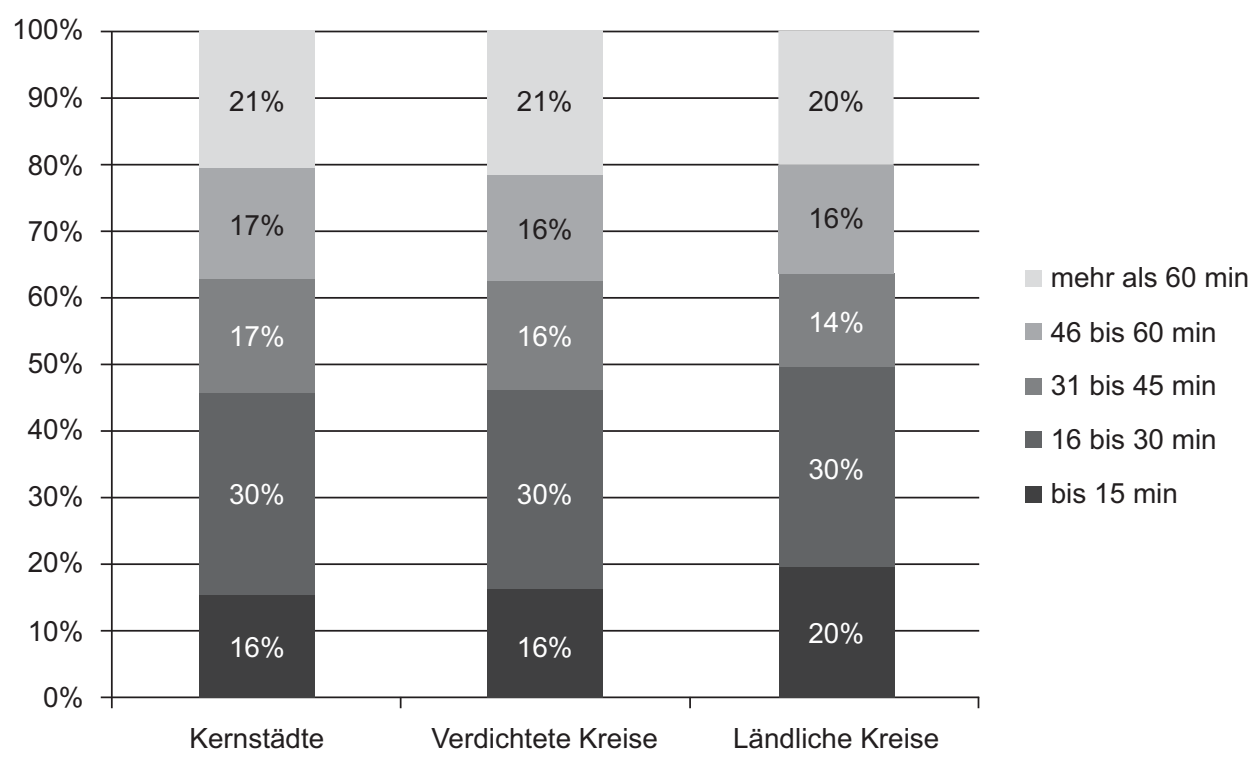

Abb. 9.2 Unterwegszeit für Arbeitswege nach Kreistypen (Datenquelle: [1])

\subsubsection{Zwischenfazit}

Vergleicht man verschiedene Varianten des Einsatzes autonomer Fahrzeuge im Carsharing, ist auf Seiten der Fahrzeugnutzer der Zugewinn an Komfort durch die Automatisierung der Fahraufgabe nicht grundsätzlich anders als beim privaten Fahrzeug. Auch im Fall von Carsharing wird die Fahrzeit frei für Beschäftigungen, die sich während der Fahrt mit einem autonomen Fahrzeug durchführen lassen. Der für das Carsharing wahrscheinlich entscheidende neuartige Nutzen entstünde bei der Zuführung des Fahrzeugs zum Nutzer und dem Entfernen des Fahrzeugs nach Abschluss des Nutzungsvorgangs.

Der Perspektive der Nutzer steht die der Betreiber gegenüber, die mit der Automatisierung des $\mathrm{Zu}$ - und Wegführens eine erhöhte Nutzungsfrequenz und Gesamtnutzungsdauer des einzelnen Fahrzeugs realisieren und damit die Rentabilität des Carsharing erhöhen könnten. Damit würden Unterschiede in der Nutzungsfrequenz, wie sie in Untersuchungen zum flexiblen Carsharing offenkundig werden, zumindest nivelliert. Derzeit steht die Nutzungsfrequenz in direktem Zusammenhang mit dem Standort, an dem das Fahrzeug verlassen wird. Hotspots in Innenstadtbereichen stehen Gebiete gegenüber, an denen die meisten dort abgestellten Fahrzeuge mehrere Stunden ungenutzt stehen (s. Abb. 9.3). Als durchschnittliche Nutzungsdauer geben Betreiber derzeit zwischen 62 und 78 Minuten pro Fahrzeug und Tag an [32]; damit ist das noch offene Potenzial einer zusätzlichen Auslastung der Fahrzeuge beträchtlich. 


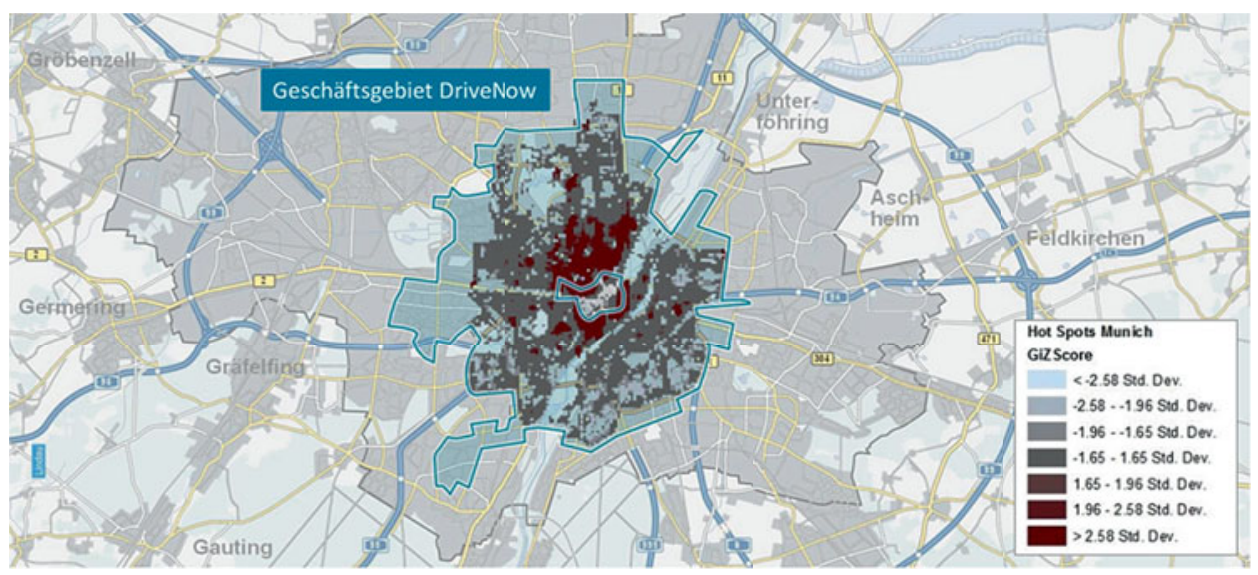

Abb. 9.3 Buchungsintensität von Fahrzeugen im flexiblen Carsharing - das Beispiel DriveNow München [14], S. 18

GIZ-Score: Ein hoher positiver Wert bedeutet, dass es in dem betreffenden Gebiet zu einer hohen Anzahl von Buchungen kommt (je höher der Wert, desto mehr Buchungen), ein niedriger Wert bedeutet, dass es nur eine geringe Anzahl von Buchungen gibt (je geringer der Wert, desto weniger Buchungen).

\subsection{Neue Mobilitätskonzepte jenseits von Carsharing: Hybridisierung des öffentlichen Verkehrs?}

Während das Carsharing, insbesondere in seiner neuen Variante als flexibles Carsharing, besondere Aufmerksamkeit erfährt, auch im Zusammenhang mit der Automatisierung der Fahrzeuge, wird oft übersehen, dass sich auch für den öffentlichen Verkehr mit der Automatisierung neue Optionen für eine (weitere) Ausdifferenzierung des derzeitigen Angebots ergeben und damit weitere Formen neuer Mobilitätskonzepte entstehen könnten. Dabei ist es unverzichtbar, auch die jeweiligen raumspezifischen Bedingungen zu berücksichtigen.

Die im Zusammenhang mit dem öffentlichen Verkehr zu diskutierenden, grundsätzlich vorhandenen Optionen betreffen

- die Neugestaltung von Intermodalität und Flexibilisierung des öffentlichen Verkehrs,

- die Individualisierung des öffentlichen Verkehrs,

- die Verdichtung der Bedienungsmöglichkeiten mit öffentlichem Verkehr.

Der besondere Vorteil, der hierbei aus dem Einsatz von autonomen Fahrzeugen entstehen würde, beträfe die bedarfsangepasste Bedienung: Fixe Routenpläne könnten durch flexible Bedienung ergänzt werden. Die zusätzlichen Routen könnten nach den Anforderungen durch die Kunden optimiert werden. Fixe Abfahrtszeiten würden durch zeit- 
lich optimierte Routenverläufe - entsprechend den Anforderungen seitens der Kunden ersetzt.

Diese Individualisierung des öffentlichen Verkehrs kommt spätestens dann einer „Hybridisierung“ gleich, wenn jenseits der Flexibilisierung von Zeiten und Routen auch Auswahlmöglichkeiten hinsichtlich der zur Verfügung stehenden Fahrzeuge angeboten werden. Grundsätzlich ist die Überlegung, den öffentlichen Verkehr über das fahrzeugspezifische Angebot zu differenzieren, nicht neu. Allerdings hat sich dies aufgrund der Kosten, die beim Vorhalten unterschiedlicher Flotten mit entsprechendem Personal entstehen, bisher nur in sehr engen, meist touristischen Nischen durchsetzen können (z. B. das Cable Car in San Francisco, der Glacier Express in der Schweiz oder der Blue Train in Südafrika).

\subsubsection{Neugestaltung von Intermodalität und Flexibilisierung des öffentlichen Verkehrs}

Intermodalität wird definiert als der Wechsel zwischen unterschiedlichen Verkehrsmitteln im Verlauf eines Weges [33]. Folgt man dieser Definition, dann gibt es - zumindest in Deutschland - intermodales Verhalten in einem nur ganz geringen Umfang. Laut der Studie „Mobilität in Deutschland 2008“ "trifft dies nur auf 1,3 Prozent aller Alltagswege zu [1]. Nicht berücksichtigt ist dabei allerdings das erhebliche $\mathrm{Ma} \beta$ an Intermodalität innerhalb des öffentlichen Verkehrs, insbesondere in der Verknüpfung von Hauptstrecken mit Zu- bzw. Abgangsstrecken. Typisches Beispiel hierfür ist der Zugang zu S-Bahnen oder Regionalbahnen über eine Buslinie, wie er vor allem in suburbanen und ländlichen Gebieten besteht. In großen Städten ist die Intermodalität zwischen den unterschiedlichen Verkehrsmitteln S-Bahn, U-Bahn, Straßenbahn und Bus angesichts der Überlagerung der Netze der einzelnen Verkehrsmittel noch deutlich stärker ausgeprägt. Im Folgenden wird vor allem die Situation in städtischen Randgebieten und weniger dicht besiedelten (ländlichen) Räumen reflektiert. Vergleichbare Szenarien für die Stadt diskutiert Heinrichs in diesem Band (s. Kap. 11).

Die Neugestaltung von Intermodalität durch die Nutzung autonomer Fahrzeuge könnte analog zum derzeit bestehenden System über den privaten Pkw oder aber ein öffentliches System stattfinden. Im Alltagsverkehr würde die Durchführung des Zu- und Abgangs für die Hauptstrecke mit dem privaten Pkw in etwa dem entsprechen, was heute als Kiss-andRide bezeichnet wird: Eine Person bringt eine andere mit dem Pkw zum Verkehrsmittel der Hauptstrecke, verabschiedet sich und nimmt dann den Pkw wieder mit, um ihn (in der Regel tagsüber) während der Abwesenheit des oder der anderen nutzen zu können. Mit einem autonomen Fahrzeug entfiele diese Notwendigkeit des „Fahrdienstes“, zumindest dann, wenn kein Verfügbarkeitsfahrer im Fahrzeug anwesend sein muss. Das Muster des Kiss-and-Ride findet sich in ähnlicher Form auch beim Fernverkehr wieder, wo übrigens heute schon Carsharing-Betreiber wie DriveNow begonnen haben, ein spezielles Angebot für den Zu- bzw. Abgang zu Flughäfen, Bahnhöfen oder Fernbus-Bahnhöfen einzurichten - beispielsweise mit reservierten Parkplätzen am Flughafen oder durch die Gewährung vergünstigter Tarife bei intermodaler Nutzung von Fernbus und Carsharing-Fahrzeug [34]. 
Wird der $\mathrm{Zu}$ - und Abgang für die Hauptstrecke mit einem öffentlichen System durchgeführt, gäbe es mittels des autonomen Fahrzeugs die Möglichkeit, wesentlich gezielter auf die Nutzerbedürfnisse einzugehen. Die festen Routen und Abfahrtzeiten könnten entfallen; vielmehr könnten Abholzeiten und Abholorte individuell vereinbart werden. Wahrscheinlich würde eine größere Flotte kleiner und mittlerer Fahrzeuge für den $\mathrm{Zu}$ - und Abgang eingesetzt; das kleinräumige System des öffentlichen Verkehrs würde zu einem System mit einer Vielzahl von Sammeltaxis mit angepasster Kapazität. Trotz Automatisierung bliebe die logistische Herausforderung enorm. Dazu kommt als wesentliche Voraussetzung für ein Funktionieren des Systems, dass die Nutzer die Vereinbarungen mit dem Betreiber mit hoher Zuverlässigkeit einhalten. Dies gälte insbesondere hinsichtlich der Abfahrtszeiten, zumal dann, wenn auf den Hauptstrecken weiterhin von einem festen Fahrplan oder doch zumindest einer Taktung auszugehen ist.

Eine solche Umgestaltung des Systems könnte auch Überlegungen zur Finanzierung der Grundversorgung mit öffentlichem Verkehr neu beleben - einerseits in Richtung eines Pay-as-you-drive, aber auch in Richtung eines pauschalisierten, über Steuern oder Abgaben pro Kopf finanzierten Angebots an alle Einwohner, wie es für Städte immer wieder diskutiert wird. Eine hohe Bediendichte auch in suburbanen und sogar ländlichen Räumen würde eine flächendeckende Pauschalabgabe rechtfertigen und könnte dabei eine Maßnahme sein, mit der die Nutzung des privaten Pkw reduziert werden kann.

\subsubsection{Individualisierung des öffentlichen Verkehrs}

Die Individualisierung des öffentlichen Verkehrs durch autonome Fahrzeuge könnte dann über die Aufhebung von im Fahrplan festgelegten Abfahrtszeiten und Routen hinausgehen, wenn es tatsächlich zu einer Reduzierung der Fahrzeuggrößen - zumindest in bestimmten Teilen des Bediengebietes - kommen würde. Damit würde sich die Möglichkeit eröffnen, den Nutzern unterschiedliche Fahrzeugtypen und Fahrzeugausstattungen anzubieten, was derzeit in einer eher rudimentären Form mit dem Angebot von 1. und 2. Klasse im öffentlichen Verkehr erfolgt, jedoch nur im Schienenverkehr tatsächlich umgesetzt ist.

Als ein möglicher Einstieg in eine solche Individualisierung des öffentlichen Verkehrs lassen sich Werksbusse interpretieren, als neueres Beispiel sei hier der mit WLAN-Zugang ausgestattete Google-Bus in und um San Francisco genannt, der Arbeitnehmer der Firma zu ihrer Arbeitsstätte bringt. In diesem Fall trifft sich eine spezifische Community in einem gemeinsamen Shuttle. Vergleichbare Konzepte, wenn auch in einer Vielfalt an weiteren Spielarten, sind denkbar und erscheinen auf der Basis autonom fahrender Fahrzeuge besonders attraktiv.

Parallel dazu ist - auch aus privater Initiative heraus - die Entwicklung neuer Car Pooling-Konzepte denkbar, die einen Mix aus Gemeinschaftsbesitz und gemeinschaftlicher, aber zeitweise auch individueller Nutzung eines Fahrzeugs darstellen könnten. Heute beschränkt sich das Car Pooling im Wesentlichen auf die mittelfristig geplante oder relativ kurzfristige Bildung von Mitfahrgemeinschaften, bei denen das Fahrzeug vom 
Eigentümer bereitgestellt wird, der gleichzeitig Fahrer ist. Auch neuere Dienste wie Uber (www.uber.com) oder Lyft (www.lyft.com) weichen von diesem Prinzip nicht ab, sie bieten aber Taxi-ähnliche Dienste an und sind somit mit den klassischen Fahrgemeinschaften, die im Alltagsverkehr überwiegend aus einer festen Gruppe an Personen bestehen, nicht zu vergleichen. Die Vermutung liegt nahe, dass mit der Einführung von autonomen Fahrzeugen diese Mitfahrdienste obsolet werden und sich hin zu Peer-to-Peer Carsharing entwickeln.

\subsubsection{Verdichtung der Bedienungsmöglichkeiten mit öffentlichem Verkehr}

In Verbindung mit dem Thema Intermodalität sind die Möglichkeiten einer dichteren Bedienung im öffentlichen Verkehr, auch in der Fläche, bereits weiter oben angesprochen worden (für den städtischen Raum s. Kap. 11). Diese aus dem Einsatz von autonomen Fahrzeugen resultierenden Vorteile gelten sowohl in räumlicher als auch in zeitlicher Hinsicht, d. h. sowohl für Räume an der Peripherie als auch für Randzeiten. Allerdings gilt auch hier - selbst unter Beachtung der Tatsache, dass Personalkosten für den Fahrer entfallen - eine wirtschaftliche Untergrenze, die sich aus der Nutzungsfrequenz ergibt. Das heißt auch, dass eine räumlich stark streuende Nutzung nur bis zu einem gewissen Umfang durch das Vorhalten einer größeren Flotte aufgefangen werden kann. In jedem Fall müssen sich auch die Investitions- und Betriebskosten für diese Fahrzeuge amortisieren.

\subsection{Implementierung von neuen Mobilitätskonzepten mit autonomen Fahrzeugen}

Carsharing erregt derzeit nicht nur Aufsehen, weil neue Formen entwickelt werden, seine Sichtbarkeit deutlich zunimmt und die Zahl der Carsharing-Nutzer in den letzten beiden Jahren sprunghaft angewachsen ist, sondern auch, weil sich abzuzeichnen scheint, dass Carsharing, das in seiner kommerziellen oder von Vereinen getragenen Variante vom privaten Autobesitz unabhängig ist, ein geeignetes Vehikel werden könnte, um neue Fahrzeugtechnologien in den Markt einzuführen. Die Nutzer erhalten entsprechende Fahrzeuge über die Flotte ihres Anbieters und können sie - ohne Aufpreis gegenüber den herkömmlichen Fahrzeugen - ausprobieren. Tatsächlich geschieht dies derzeit im Umfeld von Elektromobilität, wo Unternehmen wie DriveNow und Car2Go, aber auch Citroën mit dem MulticityAuto, Elektrofahrzeuge in ihre Carsharing-Flotte eingliedern. Seitens der Nutzer wird dies ausgesprochen positiv aufgenommen. Dazu berichten Projekte zum elektromobilen Carsharing zweierlei: Zum einen gelingt es, die neue Technologie zügig für einen großen Teil der Carsharing-Nutzer bereitzustellen und damit auch ihre Nutzung zu initiieren. Zum anderen suchen viele Nutzer auch ganz gezielt die über das Carsharing gegebene Möglichkeit, die neue Technologie zu testen und zu nutzen ([13], S.15; [15], S.19). 
Schwieriger könnte die Einführung von autonomen Fahrzeugen im öffentlichen Verkehr werden, wenngleich die bisherigen Erfahrungen mit selbstfahrenden Schienenfahrzeugen derzeit weitestgehend positiv ausfallen, so z. B. bei der Pariser Metro-Linie 1, die seit 2012 vollautomatisiert ist [36], oder auch im Fall der Nürnberger U-Bahn zum Flughafen [37]. Allerdings gelten angesichts der räumlichen Abgrenzung des Bahnkörpers hier andere Bedingungen, als dies aller Voraussicht nach im Straßenverkehr der Fall wäre. Würde auch im Straßenverkehr das autonome Fahrzeug an eine feste Infrastruktur gebunden, würden daraus nicht nur erhebliche Kosten entstehen, sondern auch die Möglichkeiten zur Flexibilisierung von Routenführungen entfallen. Besonders wichtig erscheint deshalb der testweise Einsatz von autonomen Fahrzeugen in festgelegten, kleineren öffentlichen oder halböffentlichen Bereichen, wie er in Kap. 10 beschrieben wird. Eine vergleichbar offene „Experimentier-Philosophie“ lässt sich derzeit in Deutschland nicht feststellen.

\section{$9.8 \quad$ Fazit}

Die Weiterentwicklung von Carsharing-Systemen und die Veränderung des öffentlichen Verkehrs durch den Einsatz von autonomen Fahrzeugen erscheinen grundsätzlich möglich und an vielen Stellen auch mit einem eindeutig definierbaren Nutzen für die Verkehrsteilnehmer verbunden. Im Carsharing scheint der Einsatz eines vollautomatisierten Bring- und Hol-Dienstes im Sinne von Valet-Parken fast eine logische und notwendige Konsequenz zu sein, wenn mittelfristig Verfügbarkeit und Nutzung von Carsharing noch stärker in die Fläche gehen sollen.

Daneben ist heute schon absehbar, dass rund um Carsharing und Mitfahrdienste zahlreiche neue Ideen entstehen und ausgetestet werden, die in Verbindung mit einem autonomen Fahrzeug ein noch deutlich größeres Potenzial entwickeln könnten. So werden Mitfahrdienste entwickelt, die zusätzlich Betreuungscharakter besitzen, nicht nur für ältere Menschen, sondern auch für Kinder - wie dies derzeit Boost by Mercedes-Benz in Palo Alto (Kalifornien) vormacht. Auch hier zeigt sich die unmittelbare Verknüpfung von Mobilität und Informations- und Kommunikationstechnologien als Grundlage für die Organisation des Dienstes [38].

Völlig offen ist momentan die Frage der Kosten bzw. der Rentabilität; möglicherweise sollte diese Frage im Zusammenhang mit der Finanzierung des Systems gestellt werden. Auf Seiten der Nutzer muss sich ebenfalls erst noch zeigen, ob und inwieweit das Pay-asyou-drive über das Carsharing hinaus Akzeptanz findet. Auch an dieser Stelle wird erst experimentiert. So setzt beispielsweise Spotcar in seiner aktuellen Version der zeitabhängigen Abrechnung von Anbietern wie Car2Go und DriveNow eine kilometerabhängige Preisberechnung entgegen, um damit keine unmittelbare Kostenbelastung der Kunden für staubedingte Verzögerungen im städtischen Verkehr herbeizuführen. Im öffentlichen Verkehr beginnt derzeit erst die Pay-as-you-Drive-Ära mit Systemen wie Touch\&Travel. Möglicherweise jedoch sind die Bezahlsysteme im Carsharing und vergleichbare Systeme im öffentlichen Verkehr Wegbereiter für den Einstieg in ein hoch flexibles System. 


\section{Literatur}

1. infas, DLR Alltagsverkehr in Deutschland. Struktur - Aufkommen - Emissionen -Trends. Abschlussbericht zum Projekt „Mobilität in Deutschland 2008“. Bonn und Berlin (2010)

2. Nobis, C. Multimodale Vielfalt. Quantitative Analyse multimodalen Verkehrshandelns. Dissertation, Humboldt-Universität zu Berlin (2014)

3. Beckmann, Klaus; Klein-Hitpaß, Anne (Hrsg.): Nicht weniger unterwegs, sondern intelligenter? Neue Mobilitätskonzepte. Edition difu Berlin (2013)

4. Spiegel Online: Carsharing mit selbst fahrenden Autos: Daimler eifert Google nach. erstmals veröffentlicht am 15. Juli 2014. http://www.spiegel.de/auto/aktuell/daimler-autobauer-plant-carsharing-mit-autonom-fahrenden-smarts-a-980962.html; Zugriff am 4. September 2014. (2014)

5. BCS (Bundesverband CarSharing): Datenblatt CarSharing in Deutschland Stand 01.01.2014. http://www.carsharing.de/sites/default/files/uploads/presse/pdf/datenblatt_carsharing_in_ deutschland_stand_01.01.2014.pdf; Zugriff am 4. September 2014. (2014)

6. BCS (Bundesverband Carsharing): http://www.carsharing.de/presse/pressemitteilungen/ carsharing-boom-haelt-an; Zugriff am 4. September 2014. (2014)

7. Breindl, K. CarSharing ist auch in kleinen Städten möglich. In: Bundesverband CarSharing e.V. (Hrsg.): Eine Idee setzt sich durch. 25 Jahre CarSharing. Brühl (kölner stadt- und verkehrsverlag) S.67-76. (2014)

8. Wirtschaftswoche Online: BMW, Daimler und Bahn erwägen Carsharing-Allianz. erstmals veröffentlicht am 5. Mai 2012. http:/www.wiwo.de/unternehmen/auto/mobilitaet-bmw-daimler-und-bahn-erwaegen-carsharing-allianz/6591352.html; Zugriff am 4. September 2014. (2012)

9. Deutsche Bahn AG. 215.000 Kunden entscheiden sich für „Flinkster- Mein Carsharing“. Press information, January 2013. http://www.diebahn-online.eu/de/presse/presseinformationen/ pi_it/3216552/ubd20130124.html?start=50\&itemsPerPage=20. Zugriff am 4. September 2014

10. Hampshire, R.; Gaites, C.: Peer-to-Peer Carsharing - Market analysis and potential growth. $90^{\text {th }}$ TRB Annual Meeting, 23-27 January 2011, Washington D.C. (2011)

11. Gossen, M.: Nutzen statt Besitzen - Motive und Potenziale der internetgestützten gemeinsamen Nutzung am Beispiel des Peer-to-Peer Car-Sharing. Schriftenreihe des Instituts für ökologische Wirtschaftsforschung (IÖW) 202/12. (2012)

12. Loose, W. Wie alles anfing - CarSharing als Reaktion auf die Verkehrs- und Umweltsituation in den 90er Jahren. In: Bundesverband CarSharing e.V. (Hrsg.): Eine Idee setzt sich durch. 25 Jahre CarSharing. Brühl (kölner stadt- und verkehrsverlag) S.11-16. (2014)

13. Loose, W., Nobis, C.; Holm, B.; Bake, D.: Bestandsaufnahme und Möglichkeiten der Weiterentwicklung von Car-Sharing. Berichte der Bundesanstalt für Straßenwesen - Verkehrstechnik, No.114. (2004)

14. DLR Institut für Verkehrsforschung, BMW, Bundeswehr-Universität München, Deutsche Bahn (2014): Wirkung von E-Car-Sharing-Systemen auf Mobilität und Umwelt in urbanen Räumen. Halbzeitkonferenz, Berlin, 3. Juli 2014. (2014)

15. Öko-Institut; Institut für sozialökologische Forschung (2014): SHARE - Forschung zum neuen Carsharing. Wissenschaftliche Begleitforschung zu Car2Go. Halbzeitkonferenz, Berlin, 3. Juli 2014. (2014)

16. Lichtenberg, J.; Hanel, F.: Carsharing und ÖPNV - Nutzen für beide? Eine Analyse der Situation in Frankfurt am Main. In: Der Nahverkehr H.11/2007, S.37-41. (2007)

17. Ackermann, T.; Loose, W.; Reining, C.: Die Verknüpfung von CarSharing und ÖPNV. In: Bundesverband CarSharing e.V. (Hrsg.): Eine Idee setzt sich durch. 25 Jahre CarSharing. Brühl (kölner stadt- und verkehrsverlag) S.111-122. (2014)

18. Scholl, G.; Hirschl, B.; Tibitanzl, F.: Produkte länger und intensiver nutzen. Zur Systematisierung und ökologischen Beurteilung alternativer Nutzungskonzepte. Schriftenreihe des Instituts für ökologische Wirtschaftsforschung (IÖW) 134/98. (1998) 
19. Canzler, W. Der Öffentliche Verkehr im postfossilen Zeitalter: Sechs Thesen. In: Schwedes, O. (Hrsg.): Öffentliche Mobilität. Berlin, S.229-240. (2014)

20. Benkler, Y.: Sharing nicely: On shareable goods and the emergence of sharing as a modality of economic production. http://christmasgorilla.net/longform/benkler-sharing-nicely.html; Zugriff am 4. September 2014.(ohne Jahr)

21. Scholl, G.; Schulz, L.; Süßbauer, E.; Otto, S. Nutzen statt Besitzen - Perspektiven für ressourceneffizienten Konsum durch innovative Dienstleistungen. Ressourceneffizienz Paper 12.4, Berlin. (2010)

22. Einert, D.; Schrader, U.: Die Bedeutung des Eigentums für eine Ökologisierung des Konsums. Lehrstuhl Markt und Konsum, Forschungsbericht Nr. 36, Universität Hannover. (1996)

23. Heinrichs, H.; Grunenberg, H.: Sharing Economy - Auf dem Weg in eine neue Konsumkultur? Lüneburg, Centre for Sustainability Management. (2012)

24. Bardhi, F.; Eckhardt, G. M.: Access-based consumption: The case of Carsharing. Journal of Consumer Research, Vol. 39, S.881-898. (2012)

25. Bundesverband Informationswirtschaft, Telekommunikation und Neue Medien e.V. (BITKOM): Smartphone-Boom setzt sich 2014 ungebrochen fort. erstmals veröffentlicht am 12. Februar 2014 http://www.bitkom.org/de/presse/8477_78640.aspx; Zugriff am 4. September 2014. (2014)

26. Bundesverband Informationswirtschaft, Telekommunikation und Neue Medien e.V. (BITKOM): Jeder zweite Smartphone-Besitzer installiert zusätzliche Apps. erstmals veröffentlicht am 17. Januar 2014 http://www.bitkom.org/de/presse/8477_78640.aspx; Zugriff am 4. September 2014. (2014)

27. ARD-ZDF-Onlinestudie 2013. http://www.ard-zdf-onlinestudie.de/index.php?id=419_(2013)

28. ARD-ZDF-Onlinestudie 2013. http://www.ard-zdf-onlinestudie.de/index.php?id=392_(2013)

29. Kraftfahrtbundesamt.http://www.kba.de/DE/Statistik/Fahrzeuge/Bestand/bestand_node.html; und http://www.kba.de/DE/Statistik/Fahrzeuge/Bestand/2013/2013 b ueberblick_pdf.pdf? blob=publicationFile\&v=3. (2014)

30. Bundesanstalt für Straßenwesen (BASt): Rechtsfolgen zunehmender Fahrzeugautomatisierung. Forschung kompakt 11/12. (2012)

31. Müller, J.; Schmöller, S.; Bogenberger, K.: Empirische Datenanalyse von Free Floating Car Sharing-Systemen, HEUREKA 2014, pp. 577-590. (2014)

32. Entwicklung des Carsharings in Deutschland: DriveNow und Car2Go haben die Nase vorn. http://getmobility.de/20140816-drivenow-und-car2go-haben-die-nase-vorn/; erstmals veröffentlicht am 16. August 2014; Zugriff am 4. September 2014. (2014)

33. Chlond, B.; Manz, W.: INVERMO - Das Mobilitätspanel für den Fernverkehr. Arbeitsbericht IfV-Report Nr.00-9, Institut für Verkehrswesen, Universität Karlsruhe. (2000)

34. CarsharingNews.de: MeinFernbus und DriveNow kooperieren. http://www.carsharing-news. de/meinfernbus-drivenow-kooperation/ erstmals veröffentlicht am 6. Juni 2014; Zugriff am 4. September 2014. (2014)

35. Mein Fernbus und DriveNow kooperieren. http://www.carsharing-news.de/meinfernbus-drivenow-kooperation/ vom 6. Juni 2014; Zugriff am 13.08.2014. (2014)

36. Spiegel Online: Älteste U-Bahn-Strecke von Paris: Métro-Linie 1 fährt ohne Zugführer. http:// www.spiegel.de/reise/aktuell/aelteste-u-bahnstrecke-von-paris-metro-linie-1-faehrt-ohne-zugfuehrer-a-795718.html; erstmals veröffentlicht am 3. November 2011; Zugriff am 17. August 2014. (2011)

37. Youtube: Fahrt in der führerlosen U-Bahn in Nürnberg. https://www.youtube.com/ watch?v=uPPpIGesPTY; erstmals veröffentlicht am 10. Dezember 2012; Zugriff am 4. September 2014. (2012)

38. Boostbybenz. https://boostbybenz.com/; Zugriff am 4. September 2014. (2014) 